\title{
Patients presenting to the hospital with MRSA pneumonia: differentiating characteristics and outcomes with empiric treatment
}

\author{
Emi Minejima ${ }^{1}$, Mimi Lou' ${ }^{1}$ Paul Nieberg ${ }^{2}$ and Annie Wong-Beringer ${ }^{1,2^{*}}$
}

\begin{abstract}
Background: Concern for MRSA in patients presented to the hospital with pneumonia may be overestimated leading to excessive prescribing of empiric anti-MRSA therapy. This study aims to identify at-risk patients and treatment outcomes.

Methods: Adults hospitalized during 2005-2011 with pneumonia diagnosed within $48 \mathrm{~h}$ of admission were included. Medical charts were retrospectively reviewed for relevant data. Patients with MRSA were matched 1:1 to those with non-MRSA pathogen or negative culture. A published risk scoring system for MRSA pneumonia was applied.

Results: 268 elderly patients were included, 134 patients in each group. Compared to non-MRSA group, MRSA patients presented more acutely ill ( $p$ <.0001) (pneumonia severity index score, 150 vs 93; vasopressor therapy, 34\% vs 6\%; ICU admission, $47 \%$ vs $13 \%$; and mechanical ventilation, $35 \%$ vs $10 \%)$ and had worse outcomes ( $p<0.0001)$ (time to reach clinical stability, 6 vs $2.5 \mathrm{~d}$; length of stay, 10 vs 5 d; clinical failure, $28 \%$ vs 4\%; 28 -day mortality, $22 \%$ vs 3\%). When applied to our patients, a published risk scoring scheme had $93 \%$ sensitivity but lacked specificity at $55 \%$; $40 \%$ of medium-risk patients did not have MRSA. A history of MRSA infection or pneumonia differentiated the latter group. Most MRSA patients $(66 \%, 88 / 134)$ were treated empirically (primarily vancomycin) but outcome was not improved by receipt of empiric therapy.

Conclusions: Use of a published risk scoring scheme with additional variables from this study can potentially reduce overprescribing of anti-MRSA empiric therapy in patients presented to the hospital with pneumonia. Prospective studies evaluating the treatment benefit of non-vancomycin alternatives as empiric therapy are needed.
\end{abstract}

Keywords: Pneumonia, Methicillin-resistant Staphylococcus aureus, Community-onset pneumonia, Healthcare-associated pneumonia, Risk factors

\section{Background}

Recent epidemiologic investigations indicate that the prevalence of community-associated (CA)-MRSA infections is rising, though most involve skin and soft tissue infections $[1,2]$. While the actual incidence of MRSA in communityacquired pneumonia remains low, the incidence of MRSA in healthcare-associated pneumonia (HCAP) accounts for $28 \%$ of healthcare-associated cases [3] and exceeds that in all pneumonia types including ventilator-associated pneumonia (VAP) [4,5]. Patients with risk factors for HCAP

\footnotetext{
* Correspondence: anniew@usc.edu

${ }^{1}$ University of Southern California, School of Pharmacy, 1985 Zonal Ave, Los Angeles, CA 90033, USA

${ }^{2}$ Huntington Hospital, 100 W California Blvd, Pasadena, CA 91105, USA
}

constitute a heterogeneous group with varying risk for drug-resistant gram-positive versus gram-negative pathogens depending on functional status and type and extent of healthcare exposure [6,7]. Concern for MRSA in patients presenting to the hospital with pneumonia may be overestimated, leading to excessive prescribing of empiric antiMRSA therapy [5].

Shorr et al. recently published a scoring system to stratify patients presenting to the hospital with pneumonia to predict their risk for MRSA infection [8]. The risk score was developed based on a retrospective analysis of 5,975 adult patients from 62 US hospitals who had an ICD-9 code for pneumonia diagnosed within $48 \mathrm{~h}$ of admission of a bacterial etiology; of those 837 (14\%) had MRSA 
pneumonia. The study cohort was split into a development and validation cohort to create the risk score. The scoring system consisted of eight variables: age $<30$ or $>79$ years, recent hospitalization, nursing home exposure within the last 90 days, prior IV antibiotic therapy within the last 30 days, ICU admission, cerebrovascular disease prior to admission, dementia, and female with diabetes; and ranged from scores of $0-10$. The risk for MRSA is $<10 \%$ for score of 0 or 1 compared to $>30 \%$ for score of $\geq 6$. However, the study did not evaluate outcomes associated with treatment prescribed in the different risk groups. If externally validated, this scoring system has the potential to aid clinicians in targeting empiric anti-MRSA therapy based on risk for MRSA infection.

Our study aimed to evaluate patients with communityonset pneumonia defined as patients presenting to the hospital with pneumonia with or without healthcare exposure by: 1) comparing the clinical and epidemiologic characteristics of patients with MRSA versus non-MRSA pneumonia, 2) testing the utility of Shorr's scoring system in identifying patients at risk for MRSA, and 3) evaluating outcomes observed with empiric anti-MRSA therapy in high risk patients.

\section{Methods}

This was a retrospective cohort study conducted at a 625bed community-teaching hospital in Pasadena, California. The study protocol was approved by the Huntington Hospital Institutional Review Board. Patients hospitalized between January 2005 and October 2011 were screened by ICD-9 codes for pneumonia (481.X-486.X) and included if they were: 1) $\geq 18 y, 2$ ) diagnosed with pneumonia per CDC criteria [9] within $48 \mathrm{~h}$ of hospital admission, 3) had a respiratory culture taken, and 4) received $\geq 48 \mathrm{~h}$ of effective therapy. Patients with multiple organisms in the respiratory culture were excluded. Patients were split into two groups, those with the causative organism being MRSA vs a non-MRSA pathogen or a negative culture. Since there were many more non-MRSA pneumonia patients, those who met inclusion criteria were randomly selected to match the MRSA pneumonia patients by number. HCAP was defined per the 2005 Infectious Diseases Society of America's hospital-acquired pneumonia (HAP), VAP and HCAP guidelines [10].

Medical charts were reviewed to obtain relevant demographic, laboratory, radiographic, and clinical information: age; comorbid conditions; prior history of pneumonia within 1 year; history of healthcare exposure or prior antibiotic exposure within 90 days per medical records or stated in the patient history; any history of MRSA infection or colonization; presence of severe sepsis requiring vasopressor support; antibiotic therapy prescribed during the admission (timing and regimen); radiographic, laboratory, and clinical progress; culture and sensitivity results; length of stay; death within 28 days of pneumonia diagnosis; and hospital readmission within 30 days of discharge. Disease severity was assessed using Acute Physiology and Chronic Health Evaluation (APACHE) II score within 24 hours of admission and Pneumonia Severity Index (PSI). The data were recorded on a structured data collection form and entered into Microsoft Access.

\section{Data analysis}

Patients were grouped by respiratory culture results for MRSA vs non-MRSA; the latter group included those with negative cultures. Demographic and clinical variables and severity of presentation were compared to identify differentiating characteristics. Patients were stratified into high, medium, and low risk groups for MRSA using the risk scoring system by Shorr et al. [8]. Notably, history of dementia used in the scoring system was substituted in this study by presence of feeding tube and Intensive Care Unit (ICU) admission was recorded for any time during the hospital stay. Sensitivity, specificity, positive and negative predictive values were calculated to validate the risk scoring system.

The choice and timing of anti-MRSA therapy prescribed during admission was compared between groups. Outcome measures were: time to achieve clinical stability [11], clinical response at end of treatment, need for and duration of mechanical ventilation, total length of stay, 28-day mortality, and 30-day readmission. Clinical response was determined as complete if resolution or partial if improvement was noted with fever, leukocytosis, and signs and symptoms of pneumonia.

\section{Statistical analysis}

Univariate analysis was performed using Wilcoxon rank sum tests for non-parametric continuous data or independent $\mathrm{t}$-test for parametric continuous data and Fisher's exact test and chi-square test for categorical data where appropriate using GraphPad Prism v4.0 (San Diego, CA, USA) or SAS version 9.3 (SAS Institute, Cary, NC). A p value $<0.05$ denotes statistical significance. To assess for the most significant predictors for MRSA pneumonia, PSI score, residence prior to admission, presence of other infections at admission, prior antibiotic exposure within 90 days, and presence of feeding tubes were modeled in a multivariate logistic regression analysis controlling for age, APACHE II, and $\geq 3$ comorbid conditions. In order to assess the most significant predictors of death in the MRSA pneumonia group, a multivariate logistic regression analysis controlling for APACHE II score was performed with the inclusion of the following variables: receipt of empiric anti-MRSA therapy, presence of renal dysfunction, need for mechanical ventilation, and vasopressor requirement. 


\section{Results}

Patient characteristics and risk factors

A total of 1,615 patients with ICD-9 code for pneumonia were screened. Patients were excluded for diagnosis of pneumonia $>48 \mathrm{~h}$ after admission $(\mathrm{n}=128)$, incomplete medical record $(n=66),<48 \mathrm{~h}$ of antimicrobial therapy $(\mathrm{n}=99)$, did not meet CDC criteria for pneumonia $(\mathrm{n}=241)$, multiple organisms in the respiratory culture $(n=482)$ or none taken $(n=288)$. A total of 311 patients met inclusion criteria; 134 patients had MRSA pneumonia. An equal number of patients with non-MRSA pneumonia were randomly selected to match the MRSA group by number. Twenty-eight patients in the non-MRSA pneumonia group had a bacterial etiology: $P$. aeruginosa $(\mathrm{n}=7)$, Enterobacter $\operatorname{spp}(\mathrm{n}=3)$, methicillin-sensitive $S$. aureus $(\mathrm{n}=3)$, S. pneumoniae $(\mathrm{n}=3)$, Acinetobacter spp $(\mathrm{n}=2)$, S. pyogenes $(\mathrm{n}=2)$, Haemophilus spp. $(\mathrm{n}=1)$, Stenotrophomonas maltophilia $(\mathrm{n}=1)$, Legionella $(\mathrm{n}=1)$.

Overall, the study cohort was elderly with a median age of $79 \mathrm{y}$ and $48 \%$ were male. The most common comorbid condition was cardiovascular disease (66\%), followed by pulmonary diseases (32\%). Patients with MRSA vs nonMRSA pneumonia had distinct demographic and clinical characteristics compared to those with non-MRSA pneumonia (Table 1). MRSA patients were more likely to have $\geq 3$ comorbid conditions ( $64 \%$ vs $30 \%, p<0.0001)$, diabetes $(33 \%$ vs $19 \%, \mathrm{p}<0.0001)$, cerebral vascular accident (CVA) $(31 \%$ vs $7 \%, \mathrm{p}<0.0001)$, and renal disease $(16 \%$ vs $6 \%, \mathrm{p}=0.011)$ than patients with non-MRSA pneumonia. Notably, majority of the MRSA patients $(73 \%$ vs $17 \%, \mathrm{p}<0.0001)$ resided in a skilled nursing facility (SNF) prior to admission and half had enteral feeding tubes.

Healthcare exposure within 90 days and prior antibiotic exposure were significantly more prevalent in the MRSA than the non-MRSA group: $90 \%$ vs $37 \%$ and $52 \%$ vs $17 \%$, respectively $(\mathrm{p}<0.0001)$. Furthermore, prior history of pneumonia within 1 year of admission ( $42 \%$ vs $12 \%$ ) and a history of MRSA infection ( $20 \%$ vs $0.7 \%, \mathrm{p}<0.0001$; predominantly pneumonia) were almost exclusively present in the MRSA group. Notably, $41 \%$ of the MRSA group had other infections at the time of admission compared to $10 \%$ in the non-MRSA group. When the above risk factors were included in a multivariate regression model, residence in a SNF prior to admission (OR 5.30, CI 2.59-10.87) and prior antibiotic exposure within 90 days (OR 4.62, CI 2.16-9.91) were the two most significant predictors of MRSA pneumonia (Table 2).

MRSA pneumonia patients presented more acutely ill compared to the non-MRSA group $(\mathrm{p}<0.0001)$ as evident by the higher APACHE II scores and more patients in PSI class IV and V and admitted to the ICU with need for vasopressor therapy and mechanical ventilation.
Table 1 Demographics and patient characteristics

\begin{tabular}{|c|c|c|c|}
\hline Characteristics & $\begin{array}{c}\text { MRSA } \\
\text { pneumonia } \\
n=134(\%)\end{array}$ & $\begin{array}{l}\text { Non-MRSA } \\
\text { pneumonia } \\
n=134(\%)\end{array}$ & $p$ value \\
\hline \multicolumn{4}{|l|}{ Demographics } \\
\hline Age [median (IQR)] & $82(71.5,89)$ & $75.5(62,85)$ & 0.0002 \\
\hline Male & $67(50)$ & $62(46)$ & 0.62 \\
\hline \multicolumn{4}{|l|}{ Residence prior to admission } \\
\hline Home & $35(26)$ & $110(82)$ & $<0.0001$ \\
\hline SNF & $98(73)$ & $23(17)$ & $<0.0001$ \\
\hline Other hospital & $1(0.7)$ & $1(0.7)$ & 1 \\
\hline APACHE II [median (IQR)] & $15(11,20)$ & $10(7,13)$ & $<0.0001$ \\
\hline \multicolumn{4}{|l|}{ Comorbid conditions } \\
\hline Cardiovascular disease $^{a}$ & $92(69)$ & $84(63)$ & 0.37 \\
\hline Diabetes & $44(33)$ & $26(19)$ & $<0.0001$ \\
\hline Cerebrovascular accident (CVA) & $41(31)$ & $9(7)$ & $<0.0001$ \\
\hline Liver disease $^{\mathrm{b}}$ & $6(4)$ & $4(3)$ & 0.74 \\
\hline Renal disease ${ }^{c}$ & $22(16)$ & $8(6)$ & 0.011 \\
\hline Pulmonary disease ${ }^{d}$ & $47(35)$ & $39(29)$ & 0.24 \\
\hline Malignancy & $21(16)$ & $14(10)$ & 0.37 \\
\hline$\geq 3$ comorbid conditions & $86(64)$ & $40(30)$ & $<0.0001$ \\
\hline \multicolumn{4}{|l|}{ Healthcare exposure history } \\
\hline History of MRSA infection & $27(20)$ & $1(0.7)$ & $<0.0001$ \\
\hline History of MRSA pneumonia & $14(10)$ & 0 & $<0.0001$ \\
\hline $\begin{array}{l}\text { Prior healthcare exposure } \\
\text { (within } 90 \text { days) }\end{array}$ & $120(90)$ & $50(37)$ & $<0.0001$ \\
\hline $\begin{array}{l}\text { Prior antibiotic exposure } \\
\text { (within } 90 \text { days) }\end{array}$ & $70(52)$ & $23(17)$ & $<0.0001$ \\
\hline $\begin{array}{l}\text { Days since last antibiotic given } \\
\text { [median (IQR)] }\end{array}$ & $24(7,41)$ & $7(0,32)$ & 0.629 \\
\hline $\begin{array}{l}\text { Prior history of pneumonia } \\
\text { within } 1 \text { year }\end{array}$ & $56(42)$ & $16(12)$ & $<0.0001$ \\
\hline Presence of feeding tube & $67(50)$ & $15(11)$ & $<0.0001$ \\
\hline $\begin{array}{l}\text { Presence of feeding tube } \\
\text { plus history of CVA }\end{array}$ & $26(19)$ & $4(3)$ & $<0.0001$ \\
\hline \multicolumn{4}{|l|}{ Clinical presentation } \\
\hline $\begin{array}{l}\text { Presence of other infections } \\
\text { on admission }\end{array}$ & $55(41)$ & $13(10)$ & $<0.0001$ \\
\hline Admission of intensive care units & $63(47)$ & $18(13)$ & $<0.0001$ \\
\hline $\begin{array}{l}\text { Sepsis requiring vasopressor } \\
\text { support }\end{array}$ & $45(34)$ & $8(6)$ & $<0.0001$ \\
\hline Need for mechanical ventilation & $47(35)$ & $14(10)$ & $<0.0001$ \\
\hline PSI class IV or V & $123(92)$ & $71(53)$ & $<0.0001$ \\
\hline
\end{tabular}

NOTE: SNF = skilled nursing facility; PSI = Pneumonia Severity Index; APACHE = Acute Physiology and Chronic Health Evaluation; IQR = interquartile range; aHypertension, congestive heart failure, prior myocardial infarction, coronary artery disease; ${ }^{\mathrm{b}}$ Total bilirubin $>2.5 \mathrm{mg} / \mathrm{dL}^{\mathrm{C}}{ }^{\mathrm{C}} \mathrm{Scr}>2$, Chronic Kidney Disease stage 3 or worse; ${ }^{\mathrm{d}}$ Chronic obstructive pulmonary disease, emphysema, asthma. Fisher's exact or Chi-square test done for categorical variables; $p$ value $<0.05$ denotes significance.

Wilcoxon-Mann-Whitney test done for continuous variables, $\mathrm{p}$ value $<0.05$ denotes significance. 
Table 2 Risk factors predictive of MRSA pneumonia by multivariate logistic regression

\begin{tabular}{lcc}
\hline Variable & OR $(\mathbf{9 5 \%}$ Cl) & p-value \\
\hline Age & $0.995(0.970-1.021)$ & 0.7119 \\
APACHE II & $1.035(0.972-1.103)$ & 0.2833 \\
$\geq 3$ comorbid conditions & $1.947(0.975-3.888)$ & 0.0588 \\
PSI IV \& V & $3.827(1.260-11.626)$ & 0.0179 \\
Residence in SNF prior to admission & $5.301(2.585-10.870)$ & $<0.0001$ \\
Presence of other infections & $3.686(1.515-8.967)$ & 0.0040 \\
at admission & & \\
Prior antibiotic exposure within 90 days & $4.624(2.158-9.909)$ & $<0.0001$ \\
Presence of feeding tubes & $2.357(1.062-5.231)$ & 0.0350 \\
\hline
\end{tabular}

NOTE: PSI = Pneumonia Severity Index; APACHE = Acute Physiology and Chronic Health Evaluation.

\section{Utility of Shorr's risk score}

When patients were stratified by Shorr et al's risk scoring scheme, $55 \%$ of the patients with non-MRSA pneumonia were deemed to have low risk (scores 0-1) compared to 93\% of MRSA patients to have medium (64\%, scores $2-5)$ or high risk (29\%, scores 6-10). A risk score of $>1$ (medium-high risk) had a 93\% sensitivity but lacked specificity $(55 \%)$. The negative and positive predictive values were $89 \%$ and $68 \%$, respectively. Notably, $40 \%$ of the patients with a medium risk score did not have MRSA pneumonia. Additional variables that significantly differentiated the medium-risk patients with MRSA versus non-MRSA infection were: APACHE II score, PSI class IV and V, need for vasopressor therapy, presence of other infections at admission, history of MRSA infection, and prior history of pneumonia within 1 year of admission (Table 3).

\section{Treatment}

Sixty six percent (88/134) of the MRSA pneumonia group received empiric anti-MRSA therapy, most $(85 \%, 75 / 88)$ by $8 \mathrm{~h}, 11 \%(10 / 88)$ by $24 \mathrm{~h}$, and $3 \%(3 / 88)$ by $48 \mathrm{~h}$ of admission (Table 3 ). Vancomycin was the most frequently prescribed agent $(60 \%, 53 / 88)$ for the entire treatment duration; 18 patients switched from vancomycin to linezolid 96 hours after the start of therapy while 14 patients had linezolid as primary therapy. (data not shown) Patients without empiric anti-MRSA therapy had a median delay of 3 days (IQR 2, 5) before receiving anti-MRSA therapy.

Notably, 28\% (38/134) of non-MRSA patients were also prescribed empiric anti-MRSA therapy. Interestingly, $97 \%(37 / 38)$ of these patients were classified as having low (15/38) or medium risk (22/38) of MRSA pneumonia per Shorr's scoring scheme. Overprescribing of anti-MRSA therapy in non-MRSA patients could potentially be reduced from $28 \%$ to $3 \%$ if either history of MRSA infection or prior history of pneumonia within 1 year of admission is applied.

\section{Outcomes}

Patients with MRSA pneumonia had significantly worse outcomes overall: prolonged time to reach clinical stability (6d vs $2.5 \mathrm{~d}$ ) and length of stay (10d vs $5 \mathrm{~d}$ ) as well as lower rates of clinical response (67\% vs $97 \%$ ) and higher 28 -day mortality $(22 \%$ vs $3 \%)($ all $\mathrm{p}<0.0001)$ (Table 3$)$. Those classified as having low risk for MRSA $(n=83)$ had the most favorable outcome compared to those having medium $(\mathrm{n}=$ $144)$ and high $(\mathrm{n}=41)$ risk, with increasing rates of clinical failure ( $1 \%$ vs $19 \%$ vs $34 \%)$, 28 -day mortality ( $0 \%$ vs $15 \%$ vs $27 \%$ ) and 30 -day readmission (6\% vs $13 \%$ vs $15 \%$ ) respectively (Table 3 ). Time to reach clinical stability ( $2 \mathrm{~d}$ vs $5 \mathrm{~d}$ vs $7 \mathrm{~d}$ ) and length of stay for survivors (5d vs $9 \mathrm{~d}$ vs $10 \mathrm{~d}$ ) also followed the same trend when comparing low, medium and high risk patients.

Patients with MRSA pneumonia were more likely to have received empiric anti-MRSA therapy $(n=88)$ if they were sicker at presentation with higher APACHE II (18 vs 13; $\mathrm{p}<0.0001)$, PSI scores (153.4 vs $131.3, \mathrm{p}=0.0011)$, ICU admission ( $55 \%, 48 / 88$ vs $33 \%, 15 / 46, \mathrm{p}=0.02)$, and need for vasopressor therapy $(42 \%, 37 / 88$ vs $17 \%, 8 / 46$, $\mathrm{p}=0.0041)$; and if they were from a SNF $(82 \%, 72 / 88$ vs $57 \%, 26 / 46, p=0.0043$ ). Despite receipt of empiric therapy in this sicker cohort, worse outcomes were observed with a higher rate of clinical failure ( $34 \%$ vs $17 \%, \mathrm{p}=0.046)$ and 28 -day mortality ( $27 \%$ vs $11 \%, \mathrm{p}=0.29$ ) (Table 4 ). The potential benefit of empiric treatment on outcome may have been confounded by severity of pneumonia presentation. In a subgroup analysis of the sicker patients with severity scores greater than the mean of APACHE II score $\geq 18$ and PSI score $\geq 153$, outcomes did not differ with respect to length of stay, the rate of clinical failure and mortality but 30 -day readmission rate was significantly higher in the delayed treatment group ( $50 \%$ vs $6 \%, \mathrm{p}=0.02)$. By multivariate analysis controlling for underlying severity of illness as measured by APACHE II score, those who received empiric therapy had a 1.6 fold higher risk of death compared to directed therapy, although not statistically significant $(\mathrm{p}=$ 0.45 ) (Table 5). The need for vasopressor was the most significant risk factor predictive of death (OR 3.602, $\mathrm{p}=0.037)$.

Among those with MRSA pneumonia and classified as "high risk" $(\mathrm{n}=39)$, empiric anti-MRSA therapy did not reduce 28-day mortality, clinical failure, and readmission rates. However, without empiric therapy, patients had longer hospital stay $(17 \mathrm{~d}$ vs $9 \mathrm{~d}, \mathrm{p}=0.03)$ and time to reach clinical stability $(12.5 \mathrm{~d}$ vs $6 \mathrm{~d}, \mathrm{p}=0.1)$. Vancomycin was the primary agent prescribed empirically in over half of those patients (21/39). The number of remaining patients who were initiated with or switched to linezolid was too small to allow for meaningful comparisons.

\section{Discussion}

Concerns for the spread of CA-MRSA have led to widespread prescribing of empiric anti-MRSA therapy for 
Table 3 Clinical characteristics, empiric treatment, and outcomes of patients stratified by risk for MRSA

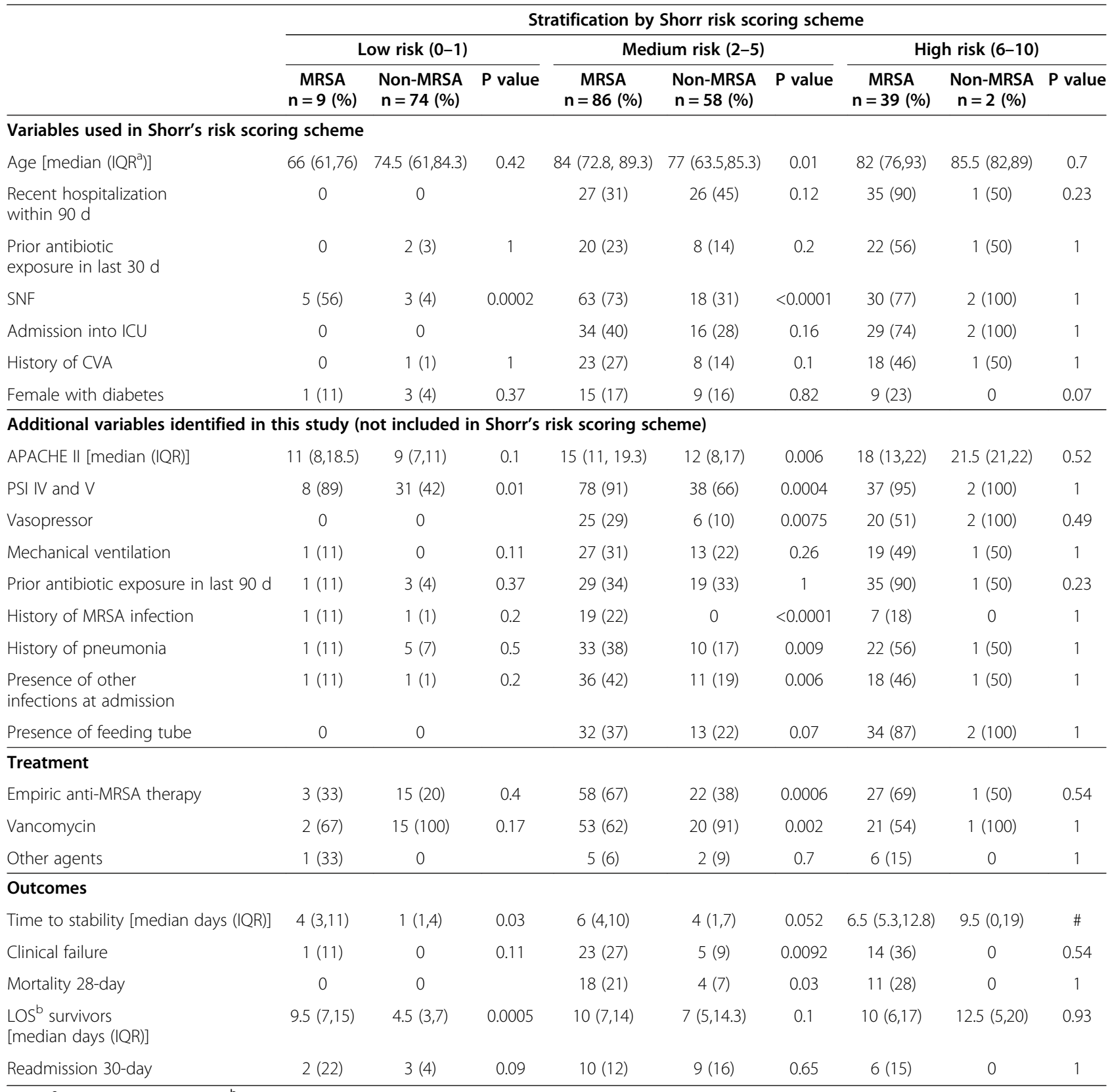

NOTE: ${ }^{~} \mathrm{IQR}=$ interquartile range; ${ }^{\mathrm{b}} \mathrm{LOS}=$ length of stay; CVA = cerebral vascular accident; SNF = skilled nursing facility; PSI = Pneumonia Severity Index; $\mathrm{APACHE}=$ Acute Physiology and Chronic Health Evaluation; \# $\mathrm{n}$ too small.

Chi-square test done for categorical variables; $p$ value $<0.05$ denotes significance.

Wilcoxon-Mann-Whitney test done for all continuous variables; $p$ value $<0.05$ denotes significance.

patients presented to the hospital with pneumonia. Therefore our study aimed to identify differentiating characteristics for MRSA pneumonia and evaluate outcomes associated with empiric anti-MRSA therapy. Our study cohort involved an elderly population who differed significantly between those with MRSA vs nonMRSA pneumonia on risk factors, severity of presentation, and outcomes. Jung et al. published a retrospective study of community-onset MRSA vs non-MRSA pneumonia in a similar patient population and found comparable rates of mortality and length of stay, though antibiotic treatment was not evaluated [12].

Shorr et al. recently published a risk score assessment to aid clinicians in identifying those at risk for MRSA among patients presenting to the hospital with pneumonia. When applied to our study cohort, the scoring scheme had a positive predictive value of $68 \%$, predicting those with nonMRSA pneumonia to have low risk and those with MRSA 


\begin{tabular}{|c|c|c|c|}
\hline Characteristics & $\begin{array}{c}\text { Empiric } \\
n=88(\%)\end{array}$ & $\begin{array}{c}\text { Directed } \\
n=46(\%)\end{array}$ & $\mathrm{p}$ value \\
\hline Age [median $\left.\left(\mathrm{IQR}^{\mathrm{a}}\right)\right]^{*}$ & $81(72.3,88)$ & $85.5(69.8,93)$ & 0.35 \\
\hline \multicolumn{4}{|l|}{ Residence prior to admission } \\
\hline Home & $15(17)$ & $20(43)$ & 0.004 \\
\hline SNF & $72(82)$ & $26(57)$ & 0.004 \\
\hline APACHE $\|$ score $[\text { mean, SD }]^{Y}$ & $17.9 \pm 6.9$ & $13.1 \pm 5.2$ & $<0.0001$ \\
\hline PSI score $\left[\right.$ mean, SD] ${ }^{Y}$ & $153.4 \pm 36.82$ & $131.3 \pm 34.9$ & 0.0011 \\
\hline Mechanical ventilation & $33(38)$ & $14(30)$ & 0.45 \\
\hline $\begin{array}{l}\text { Duration of mechanical } \\
\text { ventilation [median IQR]* }\end{array}$ & $3(2,7)$ & $6(2.5,20.75)$ & 0.064 \\
\hline Vasopressor use & $37(42)$ & $8(17)$ & 0.0041 \\
\hline \multicolumn{4}{|l|}{ Outcomes } \\
\hline $\begin{array}{l}\text { Time to reach clinical } \\
\text { stability [median (IQR)] }\end{array}$ & $7(4,10)$ & $6(3.5,11.5)$ & 0.47 \\
\hline Clinical response & $55(63)$ & $35(76)$ & 0.12 \\
\hline Mortality at 28 days & $24(27)$ & $5(11)$ & 0.029 \\
\hline Readmission within 30 days & $11(17)$ & $7(18)$ & 0.79 \\
\hline $\begin{array}{l}\text { Length of hospital stay } \\
\text { of survivors [median (IQR)]* }\end{array}$ & $10(6,15)$ & $8.5(7,14.3)$ & 0.50 \\
\hline
\end{tabular}

NOTE: ${ }^{\mathrm{a}} \mathrm{OQR}=$ interquartile range; ${ }^{\mathrm{b}} \mathrm{SD}=$ standard deviation; $\mathrm{SNF}=$ skilled nursing facility; PSI = Pneumonia Severity Index; APACHE = Acute Physiology and Chronic Health Evaluation.

Chi-square test done for categorical variables; $p$ value $<0.05$ denotes significance. *Wilcoxon-Mann-Whitney test, $\mathrm{p}$ value $<0.05$ denotes significance.

YStudent's t-test, $p$ value $<0.05$ denotes significance.

pneumonia to have high risk reasonably well. However, majority of patients fell within the medium risk group. Applying additional differentiating criteria identified from our study (e.g. history of MRSA infection or history of pneumonia within 1 year) reduced the number of non-MRSA patients in the medium risk group from $43 \%$ to $7 \%$. Also, addition of the PSI class to the scoring scheme helped capture the small number of patients with MRSA pneumonia who were "falsely" deemed to have low risk as $89 \%$ of them had PSI IV or V compared to $42 \%$ in the non-MRSA group.

Table $\mathbf{5}$ Risk factors predictive of $\mathbf{2 8 d}$ mortality in patients with MRSA pneumonia by multivariate logistic regression

\begin{tabular}{lcc}
\hline & OR $(\mathbf{9 5 \%} \mathbf{C l})$ & p value \\
\hline Receipt of empiric anti-MRSA therapy & $1.634(0.461-5.793)$ & 0.4468 \\
Renal dysfunction & $2.797(0.838-9.343)$ & 0.0946 \\
Mechanical ventilation & $2.747(0.941-8.024)$ & 0.0646 \\
Vasopressor requirement & $3.602(1.078-12.039)$ & 0.0374 \\
APACHE II score & $1.087(0.987-1.198)$ & 0.0903 \\
\hline
\end{tabular}

NOTE: APACHE = Acute Physiology and Chronic Health Evaluation.
The 2005 Infectious Diseases Society of America guideline definition of HCAP has been criticized for its lack of specificity in identifying risks for resistant pathogens [8], leading to overprescription of broad-spectrum antibiotics $[6,12,13]$. Furthermore, a study reported that guidelineadherent therapy in the management of nosocomial pneumonia, including MRSA HCAP was associated with increased mortality, hypothesized to be due to drug toxicities from aminoglycoside and colistin [13]. Over one-third (37\%) of the non-MRSA pneumonia patients were identified as HCAP by definition and $28 \%$ were given anti-MRSA therapy. By applying Shorr's scoring scheme, unnecessary prescribing of anti-MRSA therapy in low risk patients without MRSA pneumonia could have been reduced by $20 \%$. Among the $11 \%$ of "low risk" patients who had MRSA pneumonia in our cohort, outcomes were similar regardless of receipt of empiric anti-MRSA therapy.

Vancomycin was the most commonly prescribed antiMRSA therapy. Although $90 \%$ of MRSA pneumonia patients fit the definition of HCAP, the initiation rate of empiric anti-MRSA therapy was suboptimal at $68 \%$, underscoring the need to improve early recognition of at-risk patients. By using the scoring system to guide empiric therapy, an additional 30\% (40/134) of medium or high risk patients with MRSA pneumonia would have been promptly started on effective therapy. However, it is notable that a delay of 3 days in initiating anti-MRSA therapy did not lead to negative outcomes even among the subgroup of critically ill patients (APACHE II $\geq 18$ ) with more severe presentation $(n=37)$, although, the number of patients in this subgroup is small and most received vancomycin therapy. It is possible that empiric therapy with non-vancomycin alternatives (ie. linezolid or ceftaroline) may alter outcomes in this subgroup and therefore deserves to be studied further.

\section{Limitations}

This study has several limitations. First, our findings may not be applicable to all settings or patient populations due to the retrospective, single center design. We were unable to control for potentially confounding variables on outcomes such as antibiotic dosing and management of concurrent infections. MRSA grown from the respiratory culture may not represent the causative pathogen; however, we attempted to limit this possibility by including only patients who had MRSA as the sole pathogen from culture.

We included culture negative patients in the non-MRSA group in order to capture all those who presented to the hospital with pneumonia and received antibacterials for greater than 48 hours. Of the 106 patients who were culture negative in the non-MRSA group, 27/106 (25\%) received empiric anti-MRSA therapy with the median duration of 4 days (IQR 1, 5.25). Outcomes were similar between those 
with negative cultures who received empiric anti-MRSA $(\mathrm{n}=27)$ vs those did not $(\mathrm{n}=79)$ : favorable response in $25 / 27$ (93\%) vs $77 / 79$ (97\%), length of hospital stay (median: 6 vs 5 days). This data confirms that MRSA was not a likely pathogen in the culture-negative cohort and that anti-MRSA therapy was not needed in that group.

The definition of pneumonia used in this study was the CDC definition published in 2008. After our study had been completed, an updated definition was published in January 2014. Since the clinically defined pneumonia criteria remained the same and our first inclusion criteria was diagnosis of pneumonia regardless of culture results, we believe our screening criteria for inclusion into the study remains applicable. We acknowledge that the use of ICD-9 codes to identify patients can be inaccurate due to coding bias; however, we have attempted to rectify this limitation by including only patients who have met the clinical definition of pneumonia per the CDC criteria. Lastly, readmission rates could not be captured consistently as we do not have a closed healthcare system in which patients may be readmitted to the other institutions.

\section{Conclusions}

Utilization of the HCAP definition to determine patients at risk for MRSA infection leads to excessive prescribing of anti-MRSA antibiotics. The Shorr's risk scoring scheme improves identification of patients at high risk and low risk for MRSA pneumonia presenting to the hospital; however, $22 \%$ deemed to be at medium risk did not have MRSA. Based on findings from our elderly cohort, inclusion of additional variables of history of pneumonia within 1 year of admission, history of MRSA infection, and high PSI score strengthen the utility of this new scoring system. MRSA pneumonia patients present more acutely ill and have worse outcomes compared to their non-MRSA cohort. Receipt of empiric anti-MRSA therapy, primarily vancomycin, did not improve outcomes for patients with MRSA pneumonia. Prospective studies evaluating the treatment benefit of non-vancomycin alternatives as empiric therapy are needed.

\section{Competing interests}

This study was supported by an investigator-initiated research grant from Forest Pharmaceuticals to Dr. Annie Wong-Beringer.

\section{Acknowledgements}

We thank Sijy Voit, PharmD, Joyce Ho, BS, Elizabeth Plunkett, PharmD, and Levan Huang, PharmD, for their assistance in data acquisition.

Received: 14 January 2014 Accepted: 6 May 2014

Published: 10 May 2014

\section{References}

1. David MZ, Daum RS: Community-associated methicillin-resistant Staphylococcus aureus: epidemiology and clinical consequences of an emerging epidemic. Clin Microbiol Rev 2010, 23(3):616-687.

2. Klein $E$, Smith DL, Laxminarayan R: Hospitalizations and deaths caused by methicillin-resistant Staphylococcus aureus, United States, 1999-2005. Emerg Infect Dis 2007, 13(12):1840-1846.

3. Jones RN: Microbial etiologies of hospital-acquired bacterial pneumonia and ventilator-associated bacterial pneumonia. Clin Infect Dis 2010, 51(Suppl 1):S81-S87.

4. Jeffres MN, Isakow W, Doherty JA, McKinnon PS, Ritchie DJ, Micek ST, Kollef MH: Predictors of mortality for methicillin-resistant Staphylococcus aureus healthcare-associated pneumonia: specific evaluation of vancomycin pharmacokinetic indices. Chest 2006, 130(4):947-955.

5. Wunderink RG: How important is methicillin-resistant Staphylococcus aureus as a cause of community-acquired pneumonia and what is best antimicrobial therapy? Infect Dis Clin North Am 2013, 27(1):177-188.

6. Ewig S, Welte T, Chastre J, Torres A: Rethinking the concepts of community-acquired and health-care-associated pneumonia. Lancet Infect Dis 2010, 10(4):279-287.

7. Murphy CR, Quan V, Kim D, Peterson E, Whealon M, Tan G, Evans K, Meyers H, Cheung M, Lee BY, Mukamel DB, Huang SS: Nursing home characteristics associated with methicillin-resistant Staphylococcus aureus (MRSA) Burden and Transmission. BMC Infect Dis 2012, 12:269

8. Shorr AF, Myers DE, Huang DB, Nathanson BH, Emons MF, Kollef MH: A risk score for identifying methicillin-resistant Staphylococcus aureus in patients presenting to the hospital with pneumonia. BMC Infect Dis 2013, 13(1):268.

9. Horan TC, Andrus M, Dudeck MA: CDC/NHSN surveillance definition of health care-associated infection and criteria for specific types of infections in the acute care setting. Am J Infect Control 2008, 36(5):309-332.

10. Society AT, America IDSo: Guidelines for the management of adults with hospital-acquired, ventilator-associated, and healthcare-associated pneumonia. Am J Respir Crit Care Med 2005, 171(4):388-416

11. Halm EA, Fine MJ, Marrie TJ, Coley CM, Kapoor WN, Obrosky DS, Singer DE: Time to clinical stability in patients hospitalized with communityacquired pneumonia: implications for practice guidelines. JAMA 1998, 279(18):1452-1457.

12. Jung WJ, Kang YA, Park MS, Park SC, Leem AY, Kim EY, Chung KS, Kim YS, Kim SK, Chang J, Jung JY: Prediction of methicillin-resistant Staphylococcus aureus in patients with non-nosocomial pneumonia. BMC Infect Dis 2013, 13:370.

13. Kett DH, Cano E, Quartin AA, Mangino JE, Zervos MJ, Peyrani P, Cely CM, Ford KD, Scerpella EG, Ramirez JA, Improving Medicine through Pathway Assessment of Critical Therapy of Hospital-Acquired Pneumonia (IMPACT-HAP) Investigators: Implementation of guidelines for management of possible multidrug-resistant pneumonia in intensive care: an observational, multicentre cohort study. Lancet Infect Dis 2011, 11(3):181-189.

doi:10.1186/1471-2334-14-252

Cite this article as: Minejima et al:: Patients presenting to the hospital with MRSA pneumonia: differentiating characteristics and outcomes with empiric treatment. BMC Infectious Diseases 2014 14:252.

\author{
Authors' contributions \\ EM contributed to design, acquisition of data, analysis and interpretation of \\ data; drafted the submitted article; and provided final approval of the \\ version to be published. MS contributed to analysis and interpretation of \\ data; critically revised the submitted article; and provided final approval of \\ the version to be published. PN contributed to the design of the study; \\ critically revised the submitted article; and provided final approval of the \\ version to be published. AWB contributed to conception and design; to \\ analysis and interpretation of data; drafted the submitted article; and \\ provided final approval of the version to be published.
}

